\title{
Morphological changes in the enteric nervous system caused by carcinoma of the human large intestine
}

\author{
Janusz Godlewski \\ Department of Histology and Embryology, Faculty of Medicine, University of Warmia and Mazury \\ in Olsztyn, Olsztyn, Poland
}

\begin{abstract}
The innervations of the large intestine is responsible for it peristalsis and contractilibility. Investigations of the enteric nervous system in many colon diseases have revealed changes in this structure. No study has been carried out on morphological changes of the enteric nervous system in the human large intestine with carcinoma. The aim of this study was to investigate potential changes in the structure of the enteric neurons in patients with sigmoid and rectal cancer. Material for the study was obtained from patients undergoing operations due to carcinoma of the sigmoid colon and rectum. Microscopic observation of the cancerous tumor of the human large intestine revealed changes in the enteric nervous system innervating this part of the gastrointestinal tract. In the region of the enteric plexuses located close to the tumour, disruption of their correct placement and structure was observed. The changes also consisted of the disappearance of neurons and nerve fibers forming these plexuses. In the solid cancerous tumour, elements of the enteric nervous system were not present. Destruction of the enteric nervous system in the course of carcinoma of the large intestine may cause disruption of proper intestinal function and may be responsible for part of symptoms which the patients suffer.
\end{abstract}

Key words: enteric nervous system, enteric neurons, carcinoma of the large intestine

\section{Introduction}

A characteristic element which differentiates organs of the gastrointestinal tract is the appearance in their walls the enteric nervous system (ENS), a factor in local nervous regulation. The ENS functions quite independently from other parts of the nervous system. The system is composed of some plexuses in the wall of the intestine. Anatomical differences can be found in the structure of the ENS in various mammalian species. In the ENS, various types of plexuses are differentiated as regards their topography, spatial architecture, shape, size and chemical coding $[1,2,3]$. The main differences are related to the submucosal plexus [3]. They are dependent on the size of the intestine, the thickness of its walls, and, in particular, on the thickness of the submucous tissue of the intestine [4]. In small mammals, the ENS is made up of two plexuses: the submucosal plexus (Meissner's) and the myenteric plexus (Auerbach's, MP) $[3,5]$. In large mammals in

Correspondence: J. Godlewski, 10-628 Olsztyn,

ul. Bankowców 35, Poland; tel. (+48) 698694 528,

e-mail: janusz350@poczta.onet.pl addition to the Meissner plexus known as the inner submucosal plexus (ISP), there is an outer submucosal plexus (Schabadasch'a, OSP) [3]. The inner submucosal plexus is adjacent to the muscular lamina of the mucous membrane. The outer submucosal plexus is located in the submucous tissue adjacent to the circular layer of the muscular membrane of the intestine $[1,6,7]$. In humans, there is an additional plexus known as the intermediate submucosal plexus (IMSP) $[3,4,6,8]$. The intermediate submucosal plexus is better developed in the large intestine than in the small intestine $[4,6]$. The ENS influences, among others, the peristalsis of the intestine, intestinal transport of electrolytes and fluids, the supply of blood to the intestinal wall, and intestinal secretion [3].

Investigations of the large intestine in many diseases, have revealed changes in both the morphology of this system and the concentration of neuropeptides within it.

It has frequently been shown that inflammatory diseases of the intestine are associated with changes in the nervous system in humans and animals [9-13]. It has also been observed that components of the ENS are usually found, from the morphological viewpoint, in 
Table 1. Characteristics of primary and secondary antibodies used in this investigations.

\begin{tabular}{|l|c|c|c|c|}
\hline \multicolumn{1}{|c|}{ Antigen } & Code & Species & Dilution & Source \\
\hline \multicolumn{5}{|c|}{ Primary antibody } \\
\hline PGP & $13 \mathrm{C} 4$ & Mousc & $1: 2000$ & $\begin{array}{c}\text { Biogenesis, } \\
\text { UK }\end{array}$ \\
\hline \multicolumn{5}{|c|}{ Secondary antibody } \\
\hline $\begin{array}{l}\text { Conjugated with } \\
\text { FTTC goat } \\
\text { anti-mouse I } \gamma \mathrm{G}\end{array}$ & & & $1: 400$ & $\begin{array}{c}\text { Cappel, } \\
\text { USA }\end{array}$ \\
\hline
\end{tabular}

relatively good condition, despite damage to the walls. This may be accounted for by the adaptive ability of this organ in relation to the factors causing damage. However, major changes in the large intestine wall as a result of inflammation can also cause damage to nervous elements $[14,15]$.

Clinical symptoms associated with the development of carcinoma of the left half of the large intestine are generally well known. They include: changes in the character of bowel movements, constipation, diarrhoea and constipation alternately, pain, flatulence followed by obstruction of the lower part of the alimentary tract. Until now, such ailments were explained by the growing mass of the tumour causing a stiffening and narrowing of the intestine. The cancerous tumour gradually closes the lumen of the intestine, leading to complete obstruction of the alimentary tract.

A problem with research in this area is the gradual destruction of the structure of the intestine by the carcinoma tumour and the state of, or change in, various elements of the intestinal innervation. The hypothesis may be put forward that the innervation of the large intestine in the carcinoma tumour undergoes changes and these changes lead to a disruption to the motor action of the intestine. Are the clinical symptoms observed before complete blockage, a result only of the stiffening and narrowing of the intestine, or are they also a result of the state of innervation of the affected part of the large intestine? No research has been carried out so far on the morphological character of changes relating to the ENS in humans with carcinoma of the large intestine. For this reason, the purpose of this study was to investigate potential changes in the structure of ENS in patients with sigmoid colon and rectal cancer.

\section{Materials and methods}

The material for investigations was obtained from patients undergoing operations due to carcinoma of the sigmoid colon and rectum (Bioethical Commission permit No. 107/2004/II, dated 01.06.2004, Warmia and Mazury District Medical Association, Poland). Material was taken from 15 patients (nine women and six

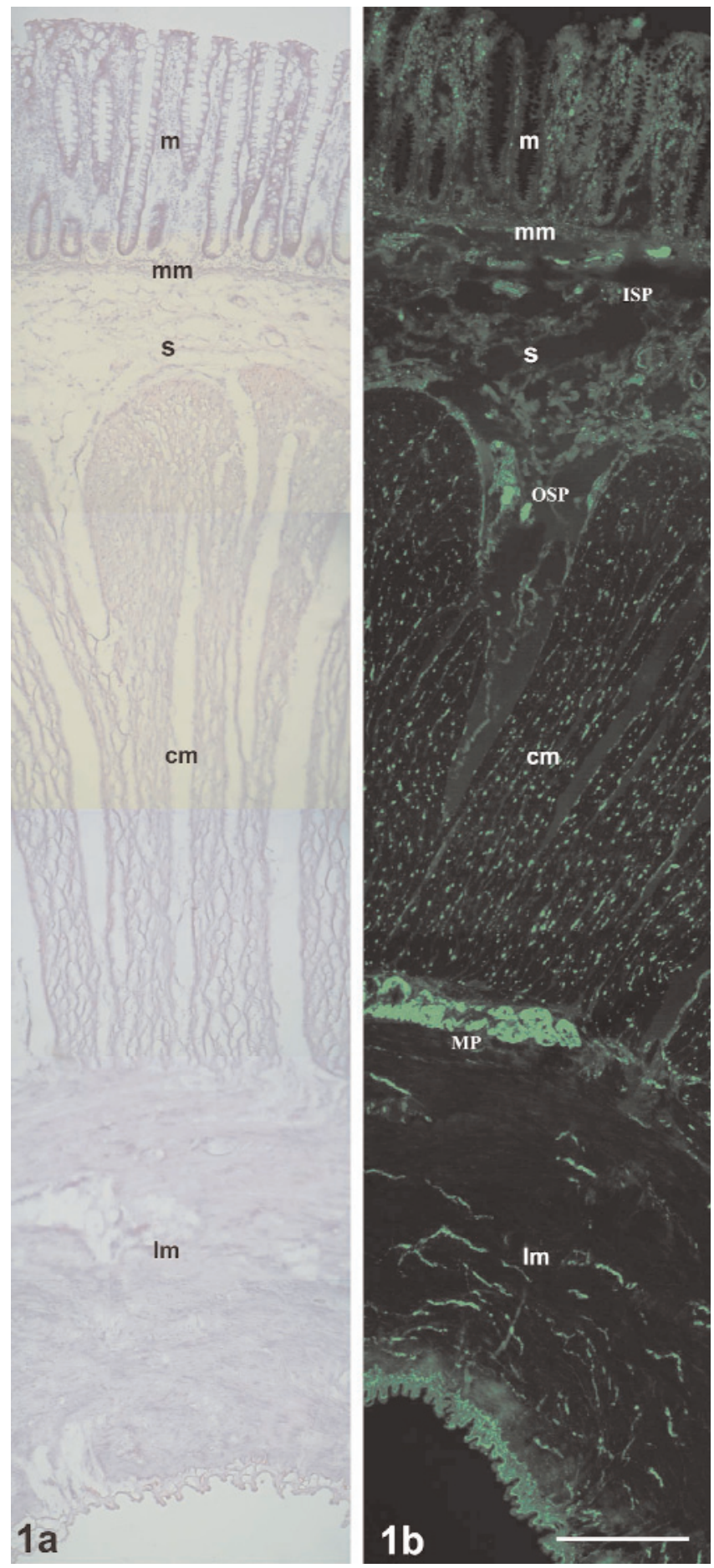

Fig. 1. a. Section through the control part of the large intestine wall. Confocal laser scanning microscope (CLSM) image showing all layers of the intestinal wall: mucous membrane (m), muscular lamina of mucous membrane $(\mathrm{mm})$, submucous tissue (s), circular layer of muscular membrane $(\mathrm{cm})$ and longitudal layer of muscular membrane $(\mathrm{lm})$. Hematoxylin-eosine staining. Bar $=300 \mu \mathrm{m}$. b. Section through the control part of the large intestine wall. CLSM image showing regular arrangement of layers of the large intestine wall with normally positioned plexuses: inner submucosal plexus (ISP), outer submucosal plexus (OSP) and myenteric plexus (MP). Immunohistochemical single-labelling for protein gene product-9.5 (PGP 9.5). $\mathrm{Bar}=300 \mu \mathrm{m}$. 

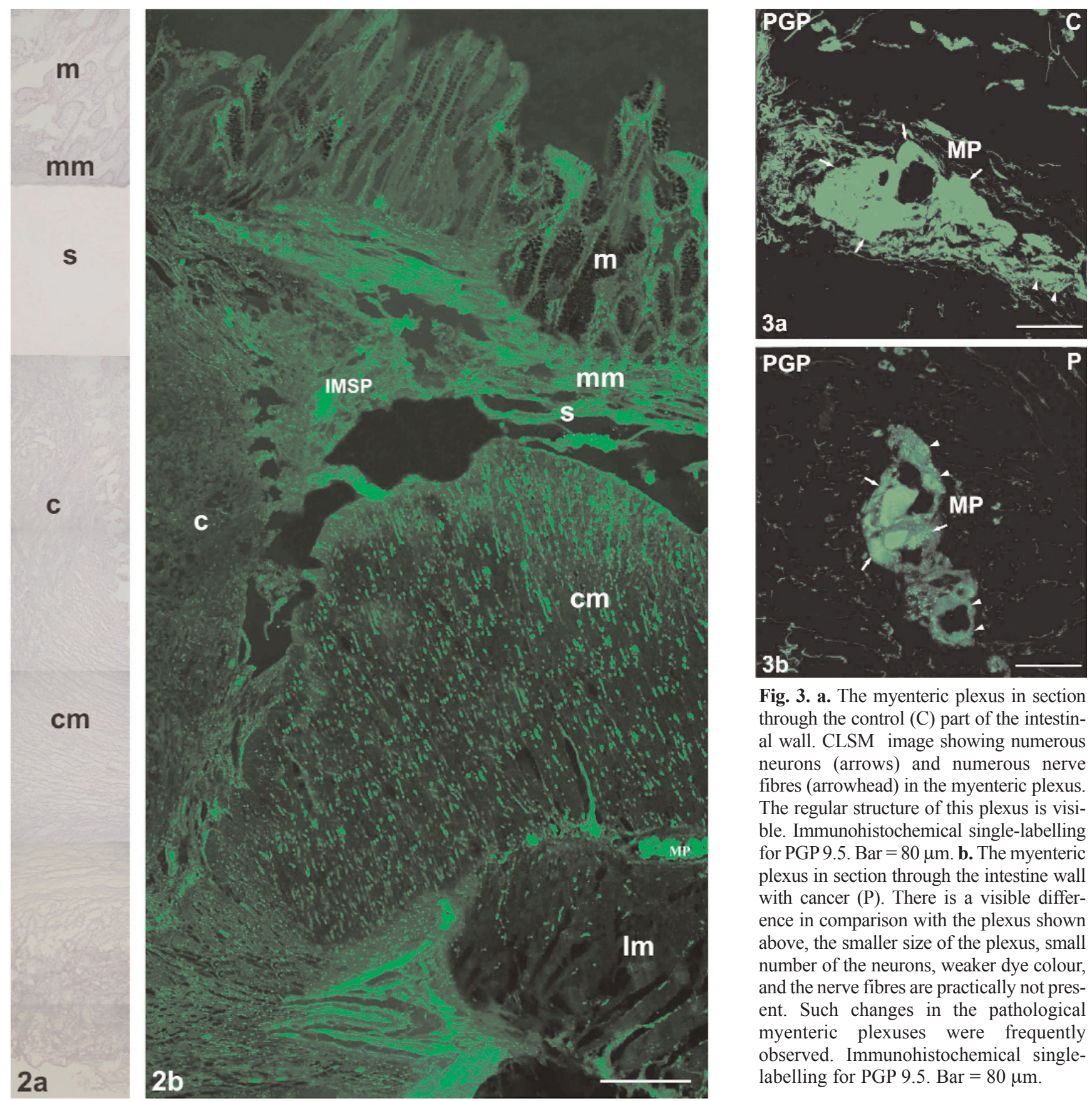

Fig. 3. a. The myenteric plexus in section through the control (C) part of the intestinal wall. CLSM image showing numerous neurons (arrows) and numerous nerve fibres (arrowhead) in the myenteric plexus. The regular structure of this plexus is visible. Immunohistochemical single-labelling for PGP 9.5. Bar $=80 \mu \mathrm{m}$. b. The myenteric plexus in section through the intestine wall with cancer $(\mathrm{P})$. There is a visible difference in comparison with the plexus shown above, the smaller size of the plexus, small number of the neurons, weaker dye colour, and the nerve fibres are practically not present. Such changes in the pathological myenteric plexuses were frequently observed. Immunohistochemical singlelabelling for PGP 9.5. Bar $=80 \mu \mathrm{m}$.

Fig. 2. a. Section through the large intestine wall with cancerous tumour (c). Hematoxylin-eosine staining. Bar $=300 \mu \mathrm{m}$. b. Section through the large intestine wall with cancerous tumour. In this CLSM image, the tumour is visible invading normal tissues and damaging layers of the intestinal wall. No nervous elements can be observed in the solid cancer tissue. The cancer is pushing the submucous plexuses through the edge of the tumour. The myenteric plexuses are damaged by the cancer. Immunohistochemical single-labelling for PGP 9.5. Bar $=300 \mu \mathrm{m}$.

men) in the period 13.07.20004 to 06.062005 . The average age was 63.0 years, the youngest patient was 41 and the oldest 78 . The tissues were obtained during scheduled operations: partial resection of the sigmoid colon and anterior resection of the rectum. Two small samples, comprising all layers of the intestinal wall, were taken, one from the large intestine showing no macroscopical changes, and the second from the neoplastic tumour of the intestine. The samples were placed for two hours in $4 \%$ buffered $(\mathrm{pH} 7.4)$ paraformaldehyde solution, then rinsed for 24 hours in phosphate buffer and preserved in $18 \%$ sucrose solution. Specimens were pre- pared for microscopic examination; cryostat sections (10 $\mu \mathrm{m}$ thick) were mounted and immunohistochemically single-labelled to reveal the presence of protein gene product-9.5 (PGP 9.5) considered to be a good panneuronal marker (Table 1).

The specimens were examined using a fluorescent microscope (Axiophot Leica, Zeiss) and a confocal microscope (Bio-Rad Microradiance MRA). Photographs of the analysed specimens were taken with the confocal microscope and scanned using appropriate program (LaserSharp 2000 v. 3.1; Bio-Rad and LaserPix v. 4.0013 Media; Cybernetics L.P.) 

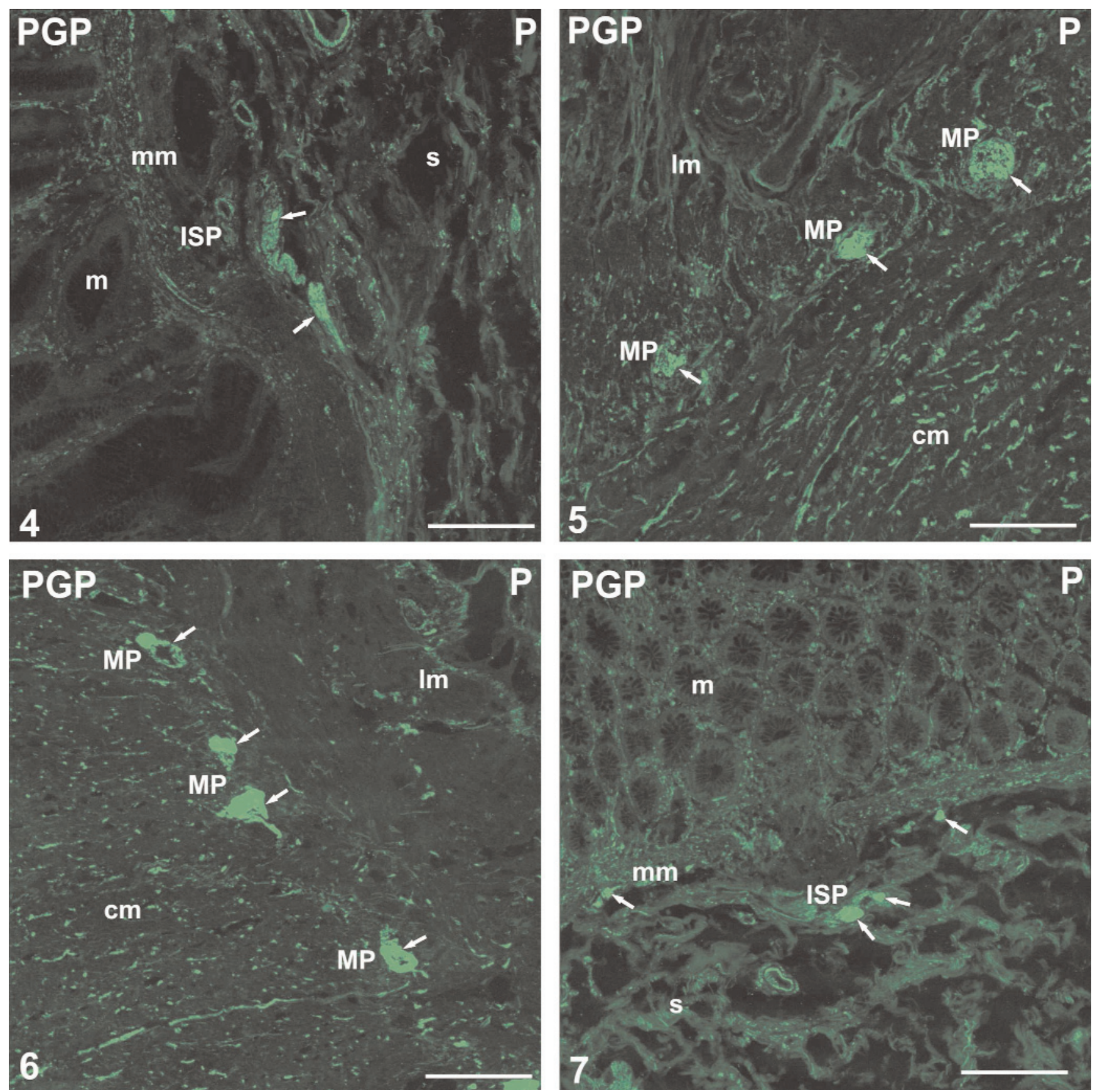

Fig. 4. Section through the mucous membrane and submucous tissue, the part of the intestinal wall with cancer. In CLSM, disruption of the myenteric lamina of the mucous membrane by the cancerous tumour is visible. The inner submucous plexus present here was dislocated by the cancer tissues. Immunohistochemical single-labelling for PGP 9.5. Bar $=200 \mu \mathrm{m}$. Fig. 5. Section through the myenteric membrane of the intestinal wall with cancer. CLSM. The still correct alignment of the myenteric plexuses is visible. The plexuses have undergone significant decay; they are very small and there contain a small numbers of neurons (arrows). Nerve fibres are very rare. Immunohistochemical singlelabelling for PGP 9.5. Bar $=200 \mu \mathrm{m}$. Fig. 6. The section through the myenteric membrane, the part of the intestine with cancer. CLSM image showing preserved correct line of the myenteric plexuses very small and contain small numbers of neurons (arrows). Immunohistochemical single-labelling for PGP 9.5. Bar $=200 \mu \mathrm{m}$. Fig. 7. Section through the mucous membrane and submucous tissue, the part of the intestinal wall with cancer. In CLSM image, breaking of the myenteric lamina of the mucous membrane by the cancer tissue is visible. The inner submucous plexus was pushed aside by the tumour. Immunohistochemical single-labelling for PGP 9.5. Bar $=200 \mu \mathrm{m}$.

\section{Results}

Microscopic observations of the specimens showed that the cancerous tumour destroyed particular layers of the large intestine wall, beginning with the mucosal membrane and submucosal tissues, and ending with the muscular membrane. It was also found that the cancerous tumour - with a sharp visible border (a type of intumescence of the expanding tumour) - caused a 
displacement of the submucosal and myenteric plexuses, and a simultaneous disappearance of their nerve fibers and neurons. Observation of other specimens with dispersed invasion of carcinoma showed that in the submucosal layer, the cancerous tissue surrounded submucosal plexuses, causing their disappearance. In the region of the muscular membrane, the dispersed type of neoplastic tumour changed the previously regular line of the myenteric plexuses, deforming them and breaking up their structure. The nerve fibers were first to disappear and next the neurons forming the myenteric plexuses. In the solid cancerous tumour which destroyed the wall of the large intestine, no elements of the enteric nervous system were found.

\section{Discussion}

As mentioned in the introduction, the ENS is decisive in the contractibility of the intestine and its peristalsis, it has an influence on the intestinal transport of electrolytes and fluids and also on the systole or diastole of the blood vessels found in the intestine wall [3]. It seems clear that morphological changes in the innervation of the intestine should have an influence on the correct functioning of the intestine.

It is acknowledged that incorrect development of the nervous system in the intestinal wall - as observed in congenital conditions such as Neuronal intestinal dysplasia is a pathophysiological cause of serious disruption of peristalsis leading to constipation [16-18]. However, in Hirshsprung's disease, congenital lack of plexuses in the large intestine leads to a pathological contraction of this section of the intestine and a consequent widening of the intestine occurs above the site of the stricture. In the widened part of the intestine, an increase in density of disorganised nerve fibers can be observed [19]. The range of investigations caried out so far indicate that in the course of inflammatory neuropathy of the intestine which leads to damage of the innervation, disruption of the peristalsis and constipation also occur. Degenerative changes in the intestinal wall together with a decrease in the number of nervous elements, and particularly neurons, in the region of the myenteric plexus have been confirmed. Such changes take place in the course of Chaga's disease, in which Trypanosoma cruzi parasites destroy the ENS, causing chronic constipation and leading to serious pathological widening of the large intestine $[18,20$ 22]. Taking into account the lack of nervous elements in the solid cancerous tumour destroying the wall of the intestine, as confirmed in this investigation, together with the results of investigations of other pathologies of the intestine, where destruction of the ENS leads to gradual constipation and widening of the colon, similar pathologic changes may be indicated as taking place also in the course of carcinoma of the large intestine.

Results of these investigations confirm damage to the innervation of the large intestine in the course of carcinoma. Confirmation of destruction of the intestinal innervation may provide the basis for morphological explanation for some of the clinical symptoms manifesting themselves as disturbed, malfunctioning action of the intestine. These observations suggest that further research in this area is required.

Acknowledgments: The author wish to thank to Professor M. Łakomy for his help and support and Mr A. Penkowski for his excellent technical assistance.

\section{References}

[1] Timmermans JP, Scheuermann DW, Stach W, Adriaensen D, Groodt-Lasseel MH. Functional morphology of the enteric nervous system with special reference to large mammals. Eur J Morphol. 1992;30:113-122.

[2] Furness JB, Young HM, Pompolo S, Bornstein JC, Kunze WA, McConalogue K. Plurichemical transmission and chemical coding of neurons in the digestive tract. Gastroenterology. 1995; 108:554-563.

[3] Timmermans JP, Adriaensen D, Cornelissen W, Scheuermann DW. Structural organization and neuropeptide distribution in the mammalian enteric nervous system, with special attention to those components involved in mucosal reflexes. Comp Biochem Physiol A Physiol. 1997;118:331-340.

[ 4] Hoyle CH, Burnstock G. Neuronal populations in the submucous plexus of the human colon. J Anat. 1989b;166:7-22.

[5] Lomax AE, Furness JB. Neurochemical classification of enteric neurons in the guinea-pig distal colon. Cell Tissue Res. 2000;302:59-72.

[ 6] Crowe R, Kamm MA, Burnstock G, Lennard-Jones JE. Peptide-containing neurons in different regions of the submucous plexus of human sigmoid colon. Gastroenterology. 1992; 102:461-467.

[7] Timmermans JP, Hens J, Adriaensen D. Outer submucous plexus: an intrinsic nerve network involved in both secretory and motility processes in the intestine of large mammals and humans. Anat Rec. 2001;262:71-78.

[ 8] Hoyle CH, Burnstock G Galanin-like immunoreactivity in enteric neurons of the human colon. J Anat. 1989a; 166:2333.

[ 9] Koch TR, Carney JA, Go VL. Distribution and quantitation of gut neuropeptides in normal intestine and inflammatory bowel diseases. Dig Dis Sci. 1987;32:369-376.

[10] Kubota Y, Petras RE, Ottaway CA, Tubbs RR, Farmer RG, Fiocchi C. Colonic vasoactive intestinal peptide nerves in inflammatory bowel disease. Gastroenterology. 1992;102: 1242-1251.

[11] Belai A, Boulos PB, Robson T, Burnstock G. Neurochemical coding in the small intestine of patients with Crohn's disease. Gut. 1997; 40:767-774.

[12] Łakomy M, Sienkiewicz W, Żmudzki J, Kaleczyc J, Wąsowicz K. Changes in the expression of some neuropeptides in the intestines and nerve ganglia during porcine dysentery. Bull Vet Inst Pulawy. 2005; 393-398.

[13] Lomax AE, Fernandez E, Sharkey KA. Plasticity of the enteric nervous system during intestinal inflammation. Neurogastroenterol Motil. 2005;17:4-15.

[14] Balemba OB, Semuguruka WD, Hay-Schmidt A, Johansen MV, Dantzer V. Vasoactive intestinal peptide and substance P- 
like immunoreactivities in the enteric nervous system of the pig correlate with the severity of pathological changes induced by Schistosoma japonicum. Int J Parasitol. 2001; 31:1503-1514.

[15] De Giorgio R, Guerrini S, Barbara G, Stanghellini V, De Ponti F, Corinaldesi R, Moses PL, Sharkey KA, Mawe GM. Inflammatory neuropathies of the enteric nervous system. Gastroenterology. 2004;126:1872-1883.

[16] Ryan DP Neuronal intestinal dysplasia. Semin Pediatr Surg. 1995;4:22-25.

[17] Giaroni C, De Ponti F, Cosentino M, Lecchini S, Frigo G. Plasticity in the enteric nervous system. Gastroenterology. 1999; 117:1438-1458.

[18] De Giorgio R, Stanghellini V, Barbara G, Corinaldesi R, De Ponti F, Tonini M, Bassotti G, Sternini C. Primary enteric neuropathies underlying gastrointestinal motor dysfunction. Scand J Gastroenterol. 2000;35:114-122.
[19] Saffrey MJ, Burnstock G. Growth factors and the development and plasticity of the enteric nervous system. J Auton Nerv Syst. 1994;49:183-196.

[20] Meneghelli UG, de Godoy RA, Macedo JF, de Oliveira RB, Troncon LE, Dantas RO. Basal motility of dilated and nondilated sigmoid colon and rectum in Chagas' disease. Arq Gastroenterol. 1982;19:127-132.

[21] Meneghelli UG Chagas' disease: a model of denervation in the study of digestive tract motility. Braz J Med Biol Res. 1985;18:255-264.

[22] Meneghelli UG. Chagasic enteropathy. Rev Soc Bras Med Trop. 2004;37:252-260.

Submitted: 16 August, 2009 Accepted after reviews: 6 December, 2009 